\title{
Implications of sensory ecology for species coexistence: biased perception links predator diversity to prey size distribution
}

\author{
Kamran Safi • Björn M. Siemers
}

Received: 19 May 2009/ Accepted: 12 September 2009/Published online: 23 December 2009

(C) The Author(s) 2009. This article is published with open access at Springerlink.com

\begin{abstract}
Inherent to sensory systems is a discrepancy between the perceived and the actual environment. We modelled prey perception in different species of echolocating bats and show that differences in sensory systems can be important for shaping the niches of animals and for structuring animal communities. We argue that sensory specialization can lower interspecific competition by making the same world appear different. We specifically raise the claim that it is important to consider the interaction of sensory bias and the distribution of (prey) resource size. Using a modeling approach we assessed the potential contribution of sensory bias for species coexistence for the example of bat echolocation. We show that even relatively small sensory differences among coexisting species can translate into significant differences in access to food resources, if prey size distribution is skewed towards small prey. Specifically, for the prey size distribution occurring most frequently in nature, differences in sensory access to resources seem large enough to relax competition and facilitate species coexistence. Interaction between sensory bias and prey size distribution in a way that enhances species coexistence may be a general phenomenon not limited to bat echolocation.
\end{abstract}

Keywords Sensory bias · Echolocation · Neuroecology $\cdot$ Sensor filtering · Niche segregation · Predator-prey interaction

K. Safi

Institute of Zoology, Zoological Society of London, Regent's Park, London NW1 4RY, UK

K. Safi $(\bowtie)$

Vogelwarte Radolfzell, Max Planck Institute for Ornithology, Schlossallee 2,

78315 Radolfzell, Germany

e-mail: ksafi@orn.mpg.de

B. M. Siemers

Sensory Ecology Group, Max Planck Institute for Ornithology,

Eberhard-Gwinner-Straße, 82319 Seewiesen, Germany

e-mail: siemers@mail.orn.mpg.de 


\section{Introduction}

Morphological distinctiveness and related ecological divergence represents one of the fundamentals in evolutionary theory; the most famous example being the beaks of the Darwin's finches (Schluter 2000). Here, we want to advocate the idea that differences in the neural and sensory system are equally important in shaping the niches of animals and structuring communities. We argue that even relatively small sensory differences among coexisting species can translate into significant differences in access to food resources, if, as typically found in nature (White et al. 2007), food size distribution is skewed. While sensory adaptations are often less conspicuous to researchers than morphological adaptations, a series of studies outline their importance for defining a species' niche (Shlaer 1972; Bernays and Wcislo 1994; Catania and Henry 2006; Dekker et al. 2006; Greiner et al. 2007). Sensory and neural adaptations increase efficiency in signal reception and transmission, but at the same time result in sensory bias on the perception of the environment (Endler 1992; Ryan and Keddy-Hector 1992; Fuller et al. 2005). Sensory bias can play a role in determining a species' diet (Barclay and Brigham 1991; Faure and Barclay 1992; Caine and Mundy 2000; Siemers and Güttinger 2006; Raine and Chittka 2007) by altering the sensory access to food and thus constitutes a mechanism that reduces interspecific competition and facilitates coexistence (Dominy and Lucas 2001; Siemers and Schnitzler 2004; Hong and Sommer 2006). Recent work has started to explore the consequences of within and between species variability in sensory performance of foraging animals for their fitness under natural conditions (Siemers and Swift 2006; Melin et al. 2007; Vogel et al. 2007).

We use echolocating predatory bats to exemplify our claim in a quantitative model. We specifically focused on the theoretically tractable scenario of aerial insectivorous bats that typically use quasi constant frequency echolocation calls when searching for prey in open space (Schnitzler and Kalko 2001; Jones and Teeling 2006). As an example, we give the frequency bands used by the European community of aerial insectivorous bats in Fig. 1. Bats provide an ideal system to realistically model the influence of sensory bias on perception and its potential influence on interspecific competition for three reasons. First, echolocation is an active sensory system (Schnitzler et al. 2003); i.e., bats "illuminate" their environment with their own calls. Species differences in call parameters and thus sensory access to prey are readily accessible. Second, bats are specious (Simmons 2005); densely packed bat assemblages make up for considerable proportions of mammalian diversity in many areas (Findley 1993; Kingston et al. 2003). Therefore, there is potential for resource partitioning mechanisms. Third, physical knowledge of sound propagation and echo generation (Mohl 1988; Crocker 1998; Stilz 2004) as well as an extensive body of literature on call production and echo perception in bats is available. The latter include quantifications of source levels (call amplitudes) in the field (Holderied and von Helversen 2003; Surlykke and Kalko 2008) and measurements of reflection properties of natural prey objects (Kober and Schnitzler 1990; Waters et al. 1995; Houston et al. 2003). We combined this knowledge to estimate prey perceptibility for a hypothetic community of bats that echolocate using different call frequencies. Several previous studies have estimated echolocation range and prey detectability as a function of call frequency for bats (e.g., Barclay and Brigham 1991; Waters et al. 1995; Holderied and von Helversen 2003; Houston et al. 2003; Jung et al. 2007). We extended these in two ways: first, we aimed at realistically modeling the abiotic environmental conditions, i.e., by integrating the complex dynamical influences of atmospheric conditions and their interaction with call frequency on sound propagation and attenuation. To achieve this, we used a randomization approach 


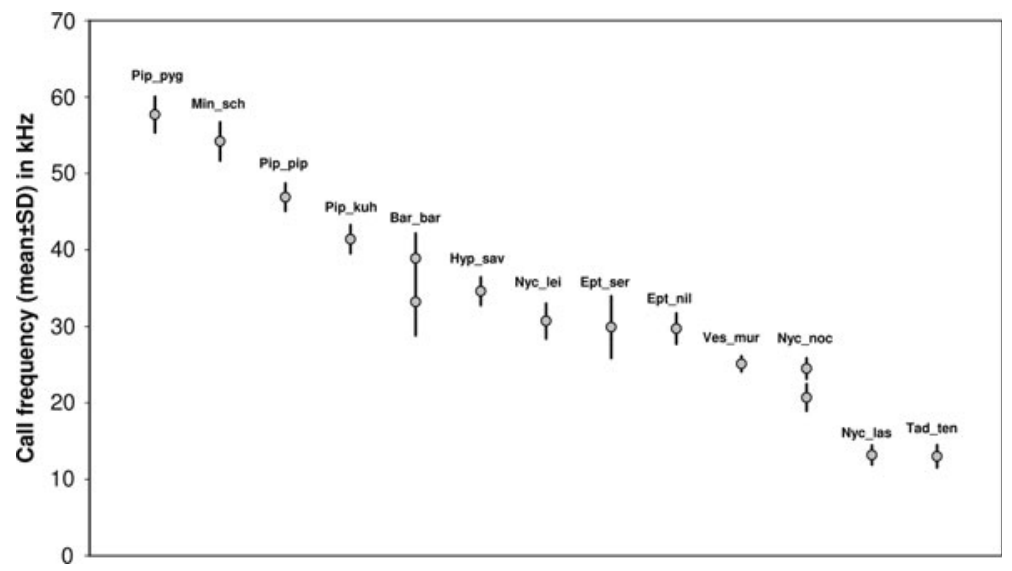

Fig. 1 Echolocation call frequencies of the European open space aerial insectivores bat community. Depicted are the frequencies of maximum amplitude and the standard deviation (except for Ept_nil where no published deviation measure could be found and thus we assumed it to be equal to the mean deviation of all other bats). The species are as follows: Bar_bar: Barbastella barbastellus; Ept_nil: Eptesicus nilssoni; Ept_ser: Eptesicus serotinus; Hyp_sav: Hypsugo savii; Min_sch: Miniopterus schreibersii; Nyc_las: Nyctalus lasiopterus; Nyc_lei: Nyctalus leisleri; Nyc_noc: Nyctalus noctula; Pip_kuh: Pipistrellus kuhlii; Pip_pip: Pipistrellus pipistrellus; Pip_pyg: Pipistrellus pygmaeus; Tad_ten: Tadarida teniotis; Ves_mur: Vespertilio murinus. Most of the data from Russo and Jones (2002) except of Ves_mur which was from Schaub and Schnitzler (2007), Ept_nil from Rydell (1990), and Nyc_las from Estók and Siemers (2009)

with a total of 1.2 billion modeled bat-prey encounters. Second, with our central question we asked how the biotic environment, e.g., prey size distribution, interacts with sensory bias. Typically, not all prey types are equally abundant. Often, abundance is skewed towards small prey (White et al. 2007). The exact relationship between abundance and the size of individuals ('individual size distribution') or species ('global or local size density relationships') depends on whether individuals or species are being counted and differs with geographic location and geographic scale (White et al. 2007). Yet, it often conforms to power functions with exponent values between -0.25 (Blackburn and Gaston 1997) and less than -1.6 (Stork and Blackburn 1993). We modeled our simulated predator community to forage in environments with different prey size distributions to investigate how and whether the interaction of sensory bias, atmospheric conditions and resource abundance influences niche overlap and thus, potentially, predator diversity.

The evolution of echolocation call frequencies is very likely a consequence of a multitude of processes, including (negative) allometric scaling of call frequency with body size (Jones 1999), phylogenetic inertia (Jones and Teeling 2006), the acoustic arms race between bats and tympanate noctuids (allotonic frequency hypothesis: Pavey and Burwell 1998; Schoeman and Jacobs 2003) and the social communication aspect of echolocation calls (Heller and von Helversen 1989; Jacobs et al. 2007). Kingston and Rossiter (2004) have provided a very interesting piece of evidence for adaptive speciation in bats by disruptive selection on call frequencies through assortative mating and perception-mediated resource partitioning. This non-withstanding, it is still unclear whether competition for food resources is crucial within bat communities (note that new techniques such as DNA barcoding from faeces for detailed diet analyses promise possible answers in the near future; Clare et al. 2009) and does drive divergence of call frequencies. We thus do not focus on the causes of call frequency differences, but rather on their evolutionary and ecological consequences. 


\section{Methods}

We estimated the perceived availability of prey for 30 hypothetical bat species as a function of their echolocation call frequency under different situations of prey abundance. The bat species in our model used constant frequency calls ranging from 5 to $150 \mathrm{kHz}$ where each species differed from the next by a $5 \mathrm{kHz}$ step.

Building on Mohl's (1988) sonar equation, we calculated the sound pressure level of a returning echo as

$$
E=\mathrm{SL}+\mathrm{TLS}+\mathrm{TLA}+\mathrm{TS}+\mathrm{RAY}+\mathrm{ANGLE}
$$

where E, energy; SL, source level; TLS, geometric attenuation; TLA, atmospheric attenuation; TS, target strength; RAY, Rayleigh scattering; ANGLE, angular attenuation; (we amended the original equation by RAY and ANGLE) to determine whether an object could have been detectable by the bat. The amount of energy that returns to the sender is primarily a function of the amount of energy which was initially emitted (source level: SL). The source level (SL) was assumed to be $100 \mathrm{~dB}$ SPL as measured $1 \mathrm{~m}$ in front of the bat (corresponding to $120 \mathrm{~dB} \mathrm{SPL}$ at $10 \mathrm{~cm}$ in front of the bat). Whether a returning echo still is detectable in turn depends on the echo perception threshold $\left(E_{\min }\right)$, which we assumed to be at $20 \mathrm{~dB}$ SPL. Judging by field measurements of SL for aerial hawking bats (Holderied and von Helversen 2003; Surlykke and Kalko 2008), and data for $E_{\min }$ (Kick 1982; Simmons et al. 1992; Jung et al. 2007) the chosen values represent a rather conservative estimate. However, our findings do not critically depend on the assumed magnitude of SL or the $E_{\min }$ nor on the "dynamic range" between them ( $80 \mathrm{~dB}$ in our case). Qualitatively, the outcome of the model remained the same for other assumed SL and $E_{\min }$ (data not shown).

After emitting a call, different processes come into play which result in the substantial loss of energy between emitting a call and receiving the echo (see e.g., Mohl 1988). First, there is "geometric attenuation" (TLS), which is the spreading loss of energy as a function of distance both on the way from the bat to the prey and back. For a target with spherical echo reflection, such as an insect,

$$
\mathrm{TLS}=40 \log _{10}\left(\frac{1}{d}\right)
$$

where $d$ is the distance of the bat to the prey. The range for $d$ was chosen to be between 1 and $6 \mathrm{~m}$. A trial with 100,000 replicates in which distance was chosen from values between 1 and $100 \mathrm{~m}$ had revealed that the maximum distance of perception was always below $6 \mathrm{~m}$ for the environmental settings and frequencies used in our model. The second factor in our equation was TLA which is the loss due to absorption of sound ("atmospheric attenuation") on the way from the bat to the prey and back again. It is given by

$$
2 \alpha(d-1) .
$$

where $\alpha$ is a function of call frequency $(f)$, airpressure $(p)$, temperature $(T)$ and relative humidity $(r)$. We followed a formula provided by Stilz (2004) for the calculation of $\alpha$. The interactions and cumulative effects of the atmospheric variables $p, T$ and $r$ on the propagation of sound are non-trivial. Therefore, we decided to randomly vary these values to estimate perceptibility of prey averaged over a range of different naturally occurring atmospheric conditions for echolocating bats in the temperate zones for each bat-prey encounter (air pressure from 84,500 to $101,500 \mathrm{~Pa}$; temperature between 5 and $18^{\circ} \mathrm{C}$, i.e., $278.15^{\circ}$ and $291.15^{\circ} \mathrm{K}$; relative humidity between 64 and $100 \%$ ). 
In addition to TLS and TLA, which are dependent on distance and atmospheric conditions, the object itself also influences the amount of returning energy by its reflection properties (TS) but also by its size relative to the frequency of the call (RAY) and its relative position to the bat (ANGLE). The target strength (TS) indicates the reflected sound intensity relative to the sound power impinging on a sonar target (Stilz 2004). In our model, prey occurred in 40 different size classes; from 1 to $40 \mathrm{~mm}$ in $1 \mathrm{~mm}$ steps. These metric prey sizes can be interpreted as body or wing size. Based on empirical measurements of insect echo reflectance (Kober and Schnitzler 1990; Waters et al. 1995; Siemers et al. 2001; Houston et al. 2003), we assumed a TS of $-70 \mathrm{~dB}$ for our smallest prey size and a TS of $-30 \mathrm{~dB}$ for the largest. We estimated TS for all intermediate prey sizes by a logarithmic interpolation:

$$
\mathrm{TS}=13.122 \log _{10}(L)-69.498,
$$

where $L$ is the size of the prey in mm. Empirical measurements suggested that TS is independent of call frequency for a wide range of frequencies (Waters et al. 1995). However, when the wavelengths $(\lambda)$ become (considerably) larger than the target $(L)$, there is increasing additional loss due to Rayleigh scattering (Kober and Schnitzler 1990; Crocker 1998). Based on measurements given in Houston et al. (2003), we approximated this additional loss (RAY) as

$$
\text { RAY }=26 \log _{10} \frac{L \frac{f}{1,000}}{343} \quad\left(\text { for } \frac{L}{\lambda}<1\right),
$$

where $f$ is the call frequency and

$$
\mathrm{RAY}=0 \quad\left(\text { for } \frac{L}{\lambda} \geq 1\right) .
$$

Finally, ANGLE is a simplified proxy for the additional loss due to prey deviating from the central axis of the bat's highly directional sonar beam and directionality of hearing. Based on directionality of a bat echolocation system, i.e., both sender and receiver characteristics combined (Grinnell and Schnitzler 1977; Schnitzler and Grinnell 1977), we assumed

$$
\mathrm{ANGLE}=-\delta\left(\frac{5}{9}\right),
$$

where $\delta$ is the angle between bat's heading and prey. Recent measurements of the bat sonar beams in the filed corroborate the assumed high directionality of echolocation (Surlykke et al. 2009). The range of $\delta$ was chosen from values between $0^{\circ}$ and $90^{\circ}$. Note that directionality of sonar beams is frequency dependent (Surlykke et al. 2009), which we neglected for the sake of simplicity. Echo detection will further be affected by call duration via forward masking and, potentially, by temporal summation of weak echoes (Schnitzler and Kalko 2001; Schnitzler et al. 2003).

We let each of the 30 hypothetical species encounter each of the 40 classes of prey $10^{6}$ times while randomly varying the environmental conditions. From these $1.2 \times 10^{9}$ encounters we calculated whether the returning echo could be perceived or not. We thus calculated the perception bias under natural circumstances in relation to the echolocation frequency and size of the prey. These data served to answer our first question (role of call frequency for biased prey perception). To address our second and central question (interaction of sensory bias with prey size distribution), we made the simulated bat 
community forage under five scenarios that differed in their resource availability. In the first scenario, all prey sizes were equally abundant (Fig. 2a). For the second, we assumed an intermediate and widely known relationship between abundance and size, abundance $=$ mass $^{-0.75}$, (Fig. 2b), and for the third a steeper size-abundance relationship with an exponent of -1.5 (Fig. 2c). Finally we also generated the inverse and thereby unnatural distributions where larger prey was more abundant than smaller prey (Fig. 2d, e).

\section{Results}

Our model corroborated the assumption that differences in call frequencies bias prey perception for echolocating bats (Fig. 2a, all prey types equally abundant). With increasing call frequency, small prey became more perceivable and large prey relatively less perceivable. The energy reflected off small echo targets increased due to shorter wave-length and thus the perceptibility for small prey increased. At the same time increasing frequencies suffered from higher atmospheric attenuation and thus the ability to detect prey at large distances decreased. The trade-off between frequency dependence of target strength (RAY) and atmospheric attenuation (TLA) resulted in perceptibility differences where bats calling at high frequencies detected small but close prey better than low-frequencyecholocating bats. The latter will perceive a prey spectrum that is sensorially skewed towards larger, distant prey (compare Barclay and Brigham 1991; Kingston and Rossiter 2004). However, the differences in perceptibility were small under the equal distribution scenario, especially for frequencies above $35 \mathrm{kHz}$ (Fig. 2a).

As an outcome of our central research question, we found sensory bias to interact strongly with prey size distribution (Fig. 2b-e). The bats' sensory differences translated into substantial increase in perceived prey availability if abundance was skewed towards small prey (Fig. 2b, c). By contrast, the perceptibility differences nearly vanished if prey distribution was skewed towards large prey (Fig. 2d, e). The model further showed that with increasing frequency the differences in perceptibility between similar echolocation frequencies became smaller. Thus, even high overabundance of small prey at some stage did not result in relevant differences in perceived prey availability. For natural prey size distribution (Fig. 2b), inter-frequency structuring of perceptibility was largest in the frequency range that most aerial hawking bats use for echolocation $(10 \mathrm{kHz}$ up to $60 \mathrm{kHz}$, red and orange lines in Fig. 2b; (Schnitzler and Kalko 2001). Compare Fig. 1 for an example of the frequency bands used by the European community of aerial hawking bats.

\section{Discussion}

Our model suggests that the interaction of sensory bias and prey size distribution can result in a reduction of interspecific competition and therefore play an important role in niche differentiation. Prey size distributions skewed towards small prey strongly amplified the call frequency dependent differences in prey detectability. They enlarged the "exclusive perception space" for the modeled bat species. The model suggests that the divergence of echolocation frequencies (Heller and von Helversen 1989; Jones and Teeling 2006) and thus the unparalleled adaptive radiation of bats might not have been such a success story, if there had not been more small insects than large ones. We reiterate that it is unclear whether competition for resources drove divergence of call frequencies. However, our data substantiate that the use of different echolocation frequencies can lead to differential 

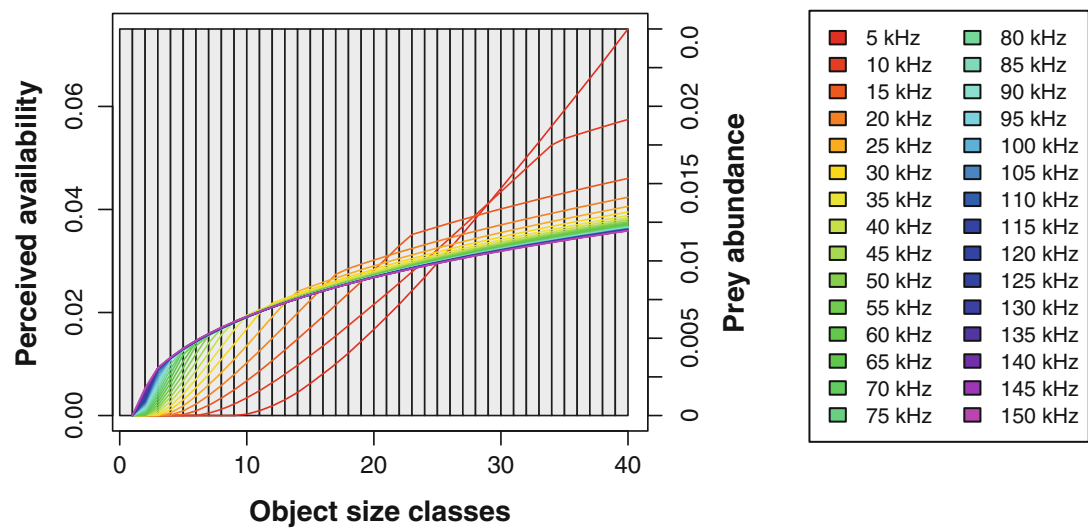

Object size classes
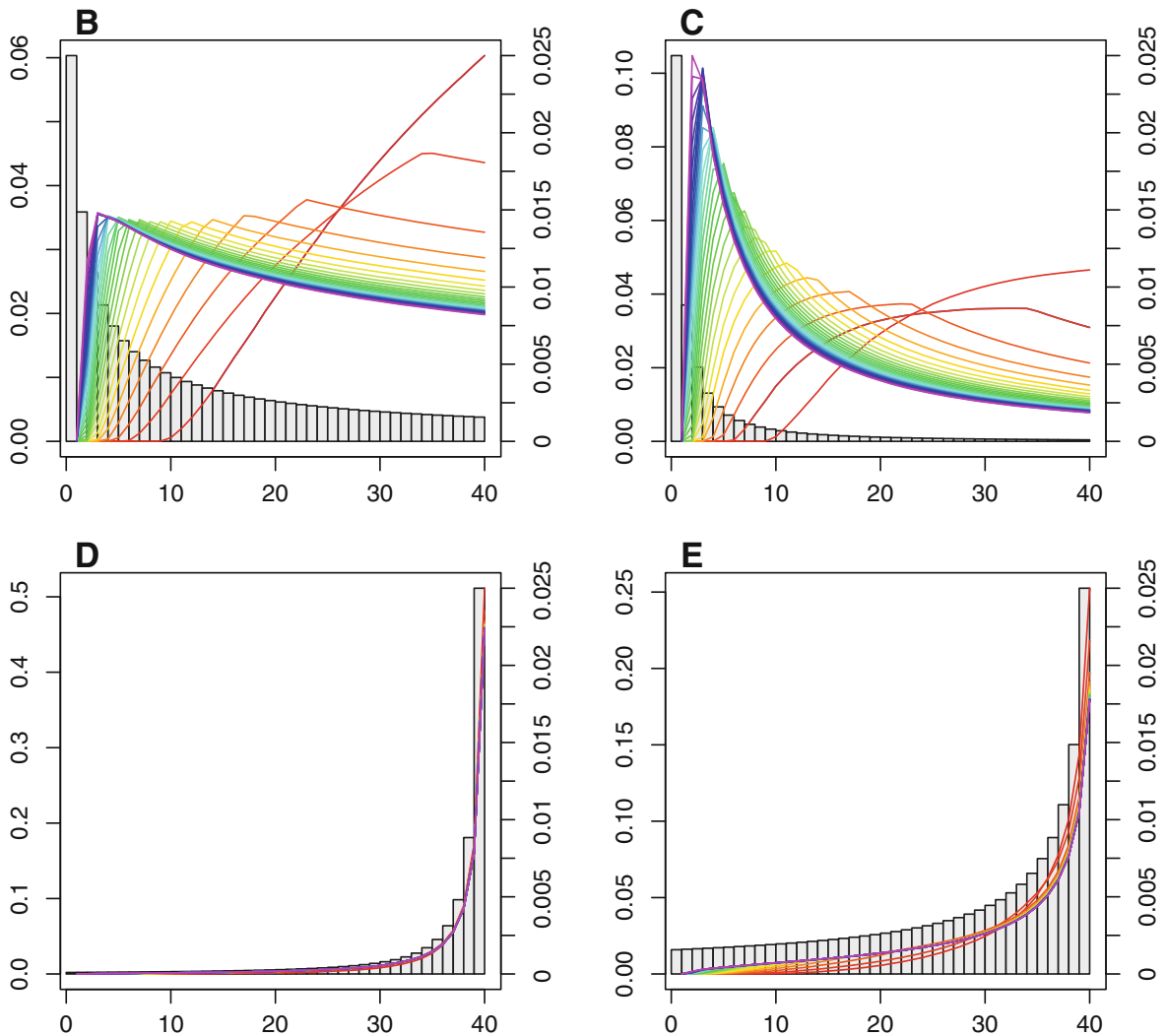

Fig. 2 Perceived availability (colored lines left y axis labels) and prey abundance (grey bars right y axes labels) for a simulated community of 30 bat species with different echolocation call frequencies (depicted in different colors) for an equal distribution of prey sizes (a) abundant small prey sizes (b and c) and abundant large prey (d and $\mathbf{e})$. All axes have the same labels as panel $A$

perception of the same environment, whatever the underlying ultimate processes might be. For example, as a consequence of negative allometric scaling of call frequency with body size it is the large bats that call low (Jones 1999) and our model suggests in line with 
Barclay and Brigham (1991) that thereby they detect large and distant prey better. This is in support of our claim that differences in the neural and sensory system are important in shaping animals' niches and structuring communities.

Currently there is only little empirical evidence for the effects of sensory bias on coexistence of predatory species. However, our model generates a series of predictions for empirical testing, once suitable data sets become available.

First, the interaction between sensory bias and prey size distribution should influence predator diversity, given that interspecific competition for food is a limiting factor. Specifically, we would expect the diversity of aerial insectivorous bats in a given region to increase with the skew of prey size distribution towards small prey. If large prey completely drop out of the local prey spectrum, then we would expect large bats to vanish and thus diversity to decrease again.

Second, we would expect that with increasing skew towards small prey, the bandwidths of call frequencies used in the respective community will spread out and specifically tend to include higher frequencies. In order to test this prediction in spatially explicit comparative studies, we will need to sample detailed information about echolocation frequencies and prey size distribution for different bat communities.

Third and in connection with the previous point, we see that sensory differences and "exclusive perceptual space" become smaller with increasing frequency. Thus, we would expect frequency spacing between coexisting species to increase with frequency. The observed echolocation frequencies used by the European open space aerial insectivorous bats nicely fit into this pattern (Fig. 1). Even overabundant small prey may not be sufficient to reduce competition between predators and compensate for the decrease in detection distance at high frequencies; i.e., there is an upper frequency limit for useful echolocation calls in aerial insectivores.

Forth, as a very speculative prediction, one might expect that bats adapt their echolocation call frequencies (within the phylogenetic and physiological limits of the species) to the local prey size distribution also at an individual level. By using different echolocation frequencies, individuals could focus their sensory channels away from each other and thus increase individual foraging efficiency. At the species level this might amount to substantial call frequency variance.

Our fifth prediction is that bat species are passively specialized on prey size by sensory bias (but see Houston et al. 2003). Such a specialization could represent an important selective pressure on insect body size evolution given the abundance and diversity of bats in many ecosystems and the huge amounts of prey that they consume (Kalka et al. 2008).

Finally, we want to emphasize that the effect of sensory bias on resource access is of course not limited to the acoustic world of echolocating bats. Differences in sensory performance within communities are probably very common throughout most sensory systems and animal taxa (Bernays and Wcislo 1994). Examples include eye size that matches the timing of foraging-bouts in ants (Greiner et al. 2007), sensitivity to scent profiles of host beetles in closely related necromenic nematodes (Hong and Sommer 2006), olfactory shifts associated with food shifts in Drosophila (Dekker et al. 2006), variation in color vision in primate communities (Dominy and Lucas 2001) and even within single primate species (Vogel et al. 2007). In weakly electric fish, electrosensory space for prey detection closely matches a species' motor volume (Snyder et al. 2007). Information on the underpinnings of these sensory differences is beginning to emerge at the level of genetics (Hong et al. 2008), neurotransmitter expression (Park et al. 2008) and receptor structure (Greiner et al. 2007). In the light of our findings on bat echolocation, it will be interesting 
to investigate how these other sensory systems may be biased, and thus may interact with different resource distribution characteristics.

Sensory variation within animal communities and species assemblages may seem minute and not as obvious as morphological differences, such as those of the beaks of the Darwin's finches. This not withstanding, we conclude from the examples we have discussed and from our model results that sensory differences do have the potential to mediate the coexistence of species given the appropriate underlying environmental conditions. We believe that sensory ecology is essential for our understanding of evolution and speciation and hope that this study will help to stimulate debate and research in this direction.

Acknowledgments We are grateful to C. Carbone, K. E. Jones, R. Page and three anonymous referees for invaluable comments and discussions on earlier versions of our manuscript. This study was supported by the Max Planck Society. KS was supported by the SNSF grant PBZHA-118824.

Open Access This article is distributed under the terms of the Creative Commons Attribution Noncommercial License which permits any noncommercial use, distribution, and reproduction in any medium, provided the original author(s) and source are credited.

\section{References}

Barclay RMR, Brigham RM (1991) Prey detection dietary niche breadth and body size in bats-why are aerial insectivorous bats so small. Am Nat 137:693-703

Bernays EA, Wcislo WT (1994) Sensory capabilities information processing and resource specialization. Q Rev Biol 69:187-204

Blackburn TM, Gaston KJ (1997) A critical assessment of the form of the interspecific relationship between abundance and body size in animals. J Anim Ecol 66:233-249

Caine NG, Mundy NI (2000) Demonstration of a foraging advantage for trichromatic marmosets (Callithrix geoffroyi) dependent on food colour. Proc R Soc Lond B Biol Sci 267:439-444

Catania KC, Henry EC (2006) Touching on somatosensory specializations in mammals. Curr Opin Neurobiol 16:467-473

Clare E, Fraser LE, Braid HE et al (2009) Species on the menu of a generalist predator the eastern red bat (Lasiurus borealis): using a molecular approach to detect arthropod prey. Mol Ecol 18:2532-2542

Crocker MJ (1998) Handbook of Acoustics. Wiley-Interscience, New York

Dekker T, Ibba I, Siju KP, Stensmyr MC, Hansson BS (2006) Olfactory shifts parallel superspecialism for toxic fruit in Drosophila melanogaster sibling D-sechellia. Curr Biol 16:101-109

Dominy NJ, Lucas PW (2001) Ecological importance of trichromatic vision to primates. Nature 410: 363-366

Endler JA (1992) Signals, signal conditions, and the direction of evolution. Am Nat 139:S125-S153

Estók P, Siemers BM (2009) Calls of a bird-eater: the echolocation behaviour of the enigmatic greater noctule Nyctalus lasiopterus. Acta Chiropterologica 11(2) (in press)

Faure PA, Barclay RMR (1992) The sensory basis of prey detection by the long-eared bat Myotis evotis and the consequences for prey selection. Anim Behav 44:31-39

Findley JS (1993) Bats: a community perspective. Cambridge University Press, Cambridge

Fuller RC, Houle D, Travis J (2005) Sensory bias as an explanation for the evolution of mate preferences. Am Nat 166:437-446

Greiner B, Narendra A, Reid SF et al (2007) Eye structure correlates with distinct foraging-bout timing in primitive ants. Curr Biol 17:R879-R880

Grinnell AD, Schnitzler HU (1977) Directional sensitivity of echolocation in the horseshoe bat Rhinolophus ferrumequinum, II. Behavioural directionality of hearing. J Comp Physiol 116:63-76

Heller KG, von Helversen O (1989) Resource partitioning of sonar frequency bands in rhinolophoid bats. Oecologia 80:178-186

Holderied MW, von Helversen O (2003) Echolocation range, wingbeat period match in aerial-hawking bats. Proc R Soc Lond B Biol Sci 270:2293-2299

Hong RL, Sommer RJ (2006) Chemoattraction in Pristionchus nematodes, implications for insect recognition. Curr Biol 16:2359-2365 
Hong RL, Witte H, Sommer RJ (2008) Natural variation in Pristionchus pacificus insect pheromone attraction involves the protein kinase EGLA-4. Proc Natl Acad Sci USA 105:7779-7784

Houston RD, Boonman A, Jones G (2003) Do echolocation signal parameters restrict bats choice of prey? In: Thomas JA, Moss CF, Vater M (eds) Echolocation in bats and dolphins. University of Chicago Press, Chicago, pp 339-345

Jacobs DS, Barclay RMR, Walker MH (2007) The allometry of echolocation call frequencies of insectivorous bats: why do some species deviate from the pattern? Oecologia 152:583-594

Jones G (1999) Scaling of echolocation call parameters in bats. J Exp Biol 202:3359-3367

Jones G, Teeling EC (2006) The evolution of echolocation in bats. Trends Ecol Evol 21:149-156

Jung K, Kalko EKV, von Helversen O (2007) Echolocation calls in Central American emballonurid bats: signal design and call frequency alternation. J Zool 272:125-137

Kalka MB, Smith AR, Kalko EKV (2008) Bats limit arthropods and herbivory in a tropical forest. Science 320:71

Kick SA (1982) Target detection by the echolocating bat Eptesicus fuscus. J Comp Physiol 145:431-435

Kingston T, Rossiter SJ (2004) Harmonic-hopping in Wallacea's bats. Nature 429:654-657

Kingston T, Francis CM, Akbar Z et al (2003) Species richness in an insectivorous bat assemblage from Malaysia. J Trop Ecol 19:67-79

Kober R, Schnitzler HU (1990) Information in sonar echoes of fluttering insects available for echolocating bats. J Acoust Soc Am 87:882-896

Melin AD, Fedigan LM, Hiramatsu C et al (2007) Effects of colour vision phenotype on insect capture by a free-ranging population of white-faced capuchins Cebus capucinus. Anim Behav 73:205-214

Mohl B (1988) Target detection by echolocating bats. In: Nachtigall PE, Moore PWB (eds) Animal sonar: processes and performance. Plenum Press, New York, pp 435-450

Park TJ, Lu Y, Juettner R et al (2008) Selective inflammatory pain insensitivity in the African naked molerat (Heterocephalus glaber). Plos Biol 6:156-170

Pavey CR, Burwell CJ (1998) Bat predation on eared moths: a test of the allotonic frequency hypothesis. Oikos 81:143-151

Raine NE, Chittka L (2007) The adaptive significance of sensory bias in a foraging context: Floral colour preferences in the bumblebee Bombus terrestris. PLoS ONE 2:e556

Russo D, Jones G (2002) Identification of twenty-two bat species (Mammalia: Chiroptera) from Italy by analysis of time-expanded recordings of echolocation calls. J Zool 258:91-103

Ryan MJ, Keddy-Hector A (1992) Directional patterns of female mate choice and the role of sensory biases. Am Nat 139:S4-S35

Rydell J (1990) Behavioural variation in echolocation pulses of the northern bat Eptesicus nilssoni. Ethology $85: 103-113$

Schaub A, Schnitzler HU (2007) Echolocation behavior of the bat Vespertilio murinus reveals the border between the habitat types "edge" and "open space". Behav Ecol Sociobiol 61:513-523

Schluter D (2000) The ecology of adaptive radiation. Oxford University Press, Oxford

Schnitzler HU, Grinnell AD (1977) Directional sensitivity of echolocation in the horseshoe bat Rhinolophus ferrumequinum I. Directionality of sound emission. J Comp Physiol 116:51-61

Schnitzler HU, Kalko EKV (2001) Echolocation by insect-eating bats. Bioscience 51:557-569

Schnitzler HU, Moss CF, Denzinger A (2003) From spatial orientation to food acquisition in echolocating bats. Trends Ecol Evol 18:386-394

Schoeman MC, Jacobs DS (2003) Support for the allotonic frequency hypothesis in an insectivorous bat community. Oecologia 134:154-162

Shlaer R (1972) An eagle's eye: quality of the retinal image. Science 176:920-922

Siemers BM, Güttinger R (2006) Prey conspicuousness can explain apparent prey selectivity. Curr Biol 16:R157-R159

Siemers BM, Schnitzler HU (2004) Echolocation signals reflect niche differentiation in five sympatric congeneric bat species. Nature 429:657-661

Siemers BM, Swift SM (2006) Differences in sensory ecology contribute to resource partitioning in the bats Myotis bechsteinii and Myotis nattereri (Chiroptera : Vespertilionidae). Behav Ecol Sociobiol 59: 373-380

Siemers BM, Stilz P, Schnitzler HU (2001) The acoustic advantage of hunting at low heights above water: behavioural experiments on the European 'trawling' bats Myotis capaccinii, M. dasycneme and M. daubentonii. J Exp Biol 204:3843-3854

Simmons NB (2005) Order chiroptera. In: Wilson DE, Reeder DM (eds) Mammal species of the world: a taxonomic and geographic reference. John Hopkins Univ Press, Baltimore, pp 312-529

Simmons JA, Moffat AJM, Masters WM (1992) Sonar gain-control, echo detection thresholds in the echolocating bat Eptesicus fuscus. J Acoust Soc Am 91:1150-1163 
Snyder JB, Nelson ME, Burdick JW, MacIver MA (2007) Omnidirectional sensory and motor volumes in electric fish. PLoS Biol 5(11):e301. doi:10.1371/journal.pbio.0050301

Stilz P (2004) Akustische Untersuchungen zur Echoortung bei Fledermäusen. Fakultät für Biologie. Universität Tübingen, Tübingen

Stork NE, Blackburn TM (1993) Abundance body-size and biomass of arthropods in tropical forest. Oikos 67:483-489

Surlykke A, Kalko EKV (2008) Echolocating bats cry out loud to detect their prey. PLoS ONE 3:e2036

Surlykke A, Pedersen SB, Jakobsen L (2009) Echolocating bats emit a highly directional sonar sound beam in the field. Proc R Soc B Biol Sci 276:853-860

Vogel ER, Neitz M, Dominy NJ (2007) Effect of color vision phenotype on the foraging of wild white-faced capuchins Cebus capucinus. Behav Ecol 18:292-297

Waters DA, Rydell J, Jones G (1995) Echolocation call design and limits on prey size-a case-study using the aerial hawking bat Nyctalus leisleri. Behav Ecol Sociobiol 37:321-328

White EP, Ernest SKM, Kerkhoff AJ, Enquist BJ (2007) Relationships between body size and abundance in ecology. Trends Ecol Evol 22:323-330 